\title{
Cardiovascular magnetic resonance at 3.0T: Current state of the art
}

\author{
John N Oshinski ${ }^{1,2^{*}}$, Jana G Delfino ${ }^{1}$, Puneet Sharma ${ }^{1}$, Ahmed M Gharib ${ }^{3}$, Roderic I Pettigrew ${ }^{3}$
}

\begin{abstract}
There are advantages to conducting cardiovascular magnetic resonance (CMR) studies at a field strength of 3.0 Telsa, including the increase in bulk magnetization, the increase in frequency separation of off-resonance spins, and the increase in $\mathrm{T} 1$ of many tissues. However, there are significant challenges to routinely performing CMR at 3.0T, including the reduction in main magnetic field homogeneity, the increase in RF power deposition, and the increase in susceptibility-based artifacts.

In this review, we outline the underlying physical effects that occur when imaging at higher fields, examine the practical results these effects have on the CMR applications, and examine methods used to compensate for these effects. Specifically, we will review cine imaging, MR coronary angiography, myocardial perfusion imaging, late gadolinium enhancement, and vascular wall imaging.
\end{abstract}

\section{Introduction}

Three Tesla (3.0T) magnetic resonance imaging (MRI) scanners have been approved for use by the United States Food and Drug Administration (US FDA) since 1999 for head imaging and since 2001 for whole body imaging. 3.0T systems have become the standard for neurological imaging at many institutions [1-3]. The adoption of 3.0T for body applications, and specifically for cardiac applications, has been somewhat slower. The slower acceptance of 3.0T for cardiac applications is due to the unique challenges posed by cardiac imaging: the requirement of a large field of view, the motion of the heart, the position of the heart within the body, the proximity of the heart to the lungs, and high radiofrequency $(\mathrm{RF})$ power deposition required in many high speed cardiac imaging sequences [4-8].

There are several advantages which motivate users to perform cardiovascular magnetic resonance (CMR) at a higher magnetic field strength. First, the bulk magnetization increases as the magnetic field strength is increased. The increased magnetization results in a theoretical increase in the signal-to-noise ratio (SNR) that is linearly related to field strength [9-11]. Second, increasing the magnetic field strength increases frequency separation of

\footnotetext{
* Correspondence: jnoshin@emory.edu

'Department of Radiology, Emory University School of Medicine, 1364 Clifton Road, Room AG34, Atlanta, GA 30322, USA

Full list of author information is available at the end of the article
}

off-resonance spins. Therefore, the frequency difference between various hydrogen-based compounds is increased. The enhanced frequency differences may be exploited for improvement in spectroscopic imaging and potentially in fat suppression. A third difference that may be seen as an advantage is that increasing the main magnetic field increases the $\mathrm{T} 1$ of many tissues, with a negligible effect on the T2 [12-16]. The increase in T1 can have beneficial effects in some applications such as myocardial tagging and myocardial perfusion sequences, but requires attention to timing parameters in other sequences, such as late gadolinium enhancement.

There are challenges to routinely performing CMR at $3.0 \mathrm{~T}$. The homogeneity of the main magnetic field $\left(\mathrm{B}_{0}\right)$ becomes more critical at 3.0T as off-resonance effects become important in many imaging sequences. The RF power deposition required for a given flip angle goes up with the square of the main magnetic field strength, so RF power deposition becomes an important consideration in imaging $[11,17]$. Additionally, maintaining the homogeneity of the field generated by the RF pulse $\left(B_{1}\right)$ is more of a challenge at 3.0T. Finally, signal loss from susceptibility-based artifacts becomes more prominent at $3.0 \mathrm{~T}$. From a practical standpoint, many implants that have been tested and deemed "MR compatible" at $1.5 \mathrm{~T}$ have not been examined at 3.0T.

The purpose of this paper is to: 1) outline the physical effects that occur when increasing the main magnetic 
field from $1.5 \mathrm{~T}$ to $3.0 \mathrm{~T}, 2$ ) outline the practical results these physical effects have on the major applications of CMR, and 3) review the technologic advances used to overcome these physical effects. Specific CMR applications to be discussed include: cine imaging, MR coronary angiography, myocardial perfusion imaging, late gadolinium enhancement, and vascular wall imaging.

\section{Background}

\section{Physical Effects of Increased Field Strength}

For the purpose of this review, we will concentrate on five major practical consequences of imaging at higher field strengths: (1) the increase in the signal-to-noise ratio, (2) the increase in the Larmor frequency, (3) the changes in relaxation times, (4) the changes in RF power deposition and specific absorption rate (SAR), and (5) the greater impact of field inhomogeneity and susceptibility.

\section{Magnetization and signal-to-noise ratio (SNR)}

Certainly, one of the greatest motivations for performing CMR at 3.0T is the increased signal-to-noise ratio (SNR) due to the increased bulk magnetization. Since many cardiac applications have relatively low SNR, the increase in SNR could enhance the clinical utility of many CMR applications and potentially enable new applications. The theoretical gain in SNR going from imaging at $1.5 \mathrm{~T}$ to $3.0 \mathrm{~T}$ is a factor of two. In many neuro-imaging applications, this increase has been realized [17]. In cardiac and body applications, the reported increases in SNR have been application and sequence dependant. Often, changes in sequence parameters are required to adapt $1.5 \mathrm{~T}$ sequences for $3.0 \mathrm{~T}$, and these changes mitigate the expected theoretical increase in SNR. This is especially true for sequences that require multiple rapid sequential RF pulses, such as steady-state free procession (SSFP) sequences. Often, the specific absorption rate (SAR) limits are exceeded at 3.0T using the parameter settings employed at $1.5 \mathrm{~T}$. To meet the SAR requirements, modifications of the sequence, such as a decrease in flip angle (which reduces SNR) or an increase in repetition time (which increases susceptibility effects) are required. Reducing the flip angle causes a "parameter-induced" reduction in SNR. Other effects, such as RF field inhomogeneity, increased susceptibility, and changes in T1 at 3.0T cause "physical-induced" reductions in SNR. These effects will be discussed in detail in the following sections.

One potential use of the increased SNR seen at 3.0T is to employ parallel imaging at 3.0T. Parallel imaging reduces imaging time by using the additional spatial encoding information provided by multiple receiver coils to reduce the number of encoding lines needed. However, the reduced imaging time decreases the SNR by a factor approximately equal to the square root of the reduction in acquisition time. For example, a reduction in scan time of 2 by parallel imaging would cause SNR to be reduced by at least a factor of or 1.4 times [18]. The increase in SNR gained at 3.0T can be used to offset this reduction in SNR seen with parallel imaging techniques. The pairing of $3.0 \mathrm{~T}$ and parallel imaging could reduce scan time by a factor of two with a preservation of SNR at $1.5 \mathrm{~T}$ values. The higher resonant frequencies at 3.0T have another potential advantage for parallel imaging. The higher frequencies allow a greater separation of the coil elements in the frequency domain. Other possible uses of the SNR increase seen at 3.0T would be to improve spatial resolution while keeping SNR values near their $1.5 \mathrm{~T}$ level, or to increase SNR above 1.5T levels while keeping total scan time constant. Larmor and RF Frequency changes

The resonant (or Larmor) frequency changes linearly with field strength. Therefore, the doubling of the magnetic field strength from $1.5 \mathrm{~T}$ to $3.0 \mathrm{~T}$ causes a doubling of the resonant frequency. The increase of the resonant frequency also increases the separation between the frequencies of individual hydrogen-based compounds. In clinical cardiac imaging, this increased separation will lead to a doubling of the frequency difference between the primary water frequency and the fat frequency. The increased difference in the fat and water frequency can cause alterations in the appearance of the so-called "India-ink" artifact at 3.0T. The India ink artifact is caused by very low signal in pixels which cross boundaries of tissues containing a combination of fat and water. These pixels experience a signal phase cancellation at certain echo times that make borders around these tissues appear dark as though they were outlined in India ink $[8,19]$. This effect is a function of the repetition time (TR) and echo time (TE) used in a particular sequence, and therefore, it may appear in sequences at $3.0 \mathrm{~T}$ when it was not seen at $1.5 \mathrm{~T}$.

The increased separation between fat and water may also allow for improvement in chemical-shift based fat suppression at $3.0 \mathrm{~T}$, as the frequency of the fat suppression pulse is farther from the primary water frequency and therefore the fat suppression pulse is less likely to inadvertently partially suppress the water signal. Chemical shift artifacts, where the spatial positions of pixels containing fat are shifted relative to water containing pixels, will be more prominent at 3.0T, however, for most cardiac sequences, the effect is negligible. The increased frequency separation between hydrogen-based compounds has significant benefits for proton spectroscopy. The increased frequency separation should create less overlap in peaks in the spectra and therefore enable better quantification of specific compound concentrations. The increase in SNR will translate to higher spectral peaks relative to the background signal $[14,15,20]$. 
The higher resonant frequency at 3.0T requires that the frequency of the RF excitation pulse change to match the 3.0T resonant frequency. At these higher resonant frequencies, significant spatial variations in the flip angle can be seen at 3.0T that are not seen at $1.5 \mathrm{~T}$. At main magnetic fields strengths of 3.0T and above, the electrical conductivity and permittivity of the body tissue as well as the shape of the body significantly affect the propagation of the magnetic fields. Additionally, the wavelength of the generated magnetic fields is on the order of the body's size. Together these effects cause the strength of RF field to vary with spatial position. The effect has been referred to as "field-focusing", because flip angles in head images are increased or "focused" near the center of the field of view. However, the effects can be quite variable and are not easily predicted, especially in cardiac imaging [9,21]. These effects can be ignored at $1.5 \mathrm{~T}$, but must be considered at $3.0 \mathrm{~T}$ and above. Using standard pulses, the resultant flip angle across the body has been reported to vary by $40 \%$ and the variations are dependant on tissue type [21-24]. Figure 1 shows a $\mathrm{CuSO}_{4}$ bottle phantom at $1.5 \mathrm{~T}$ and $3.0 \mathrm{~T}$ after a $90^{\circ} \mathrm{rf}$ pulse. Much more spatial variation of the flip angle is seen at 3.0T.

\section{Changes in RF Power and SAR}

As a direct result of increasing the main magnetic field strength, the frequency of the RF pulse increases. The increase in frequency increases the power required to create a given flip angle by the square of the change in main magnetic field $[9,11]$. The specific absorption rate (SAR) is an estimate of the time-averaged power (either per gram of tissue or over the whole body), therefore SAR of a sequence at 3.0T will have four times the SAR of the identical sequence run at $1.5 \mathrm{~T}$. The SAR limits are set to prevent excessive heating of tissue due to RF power deposition. SAR cannot be directly measured in patients, so scanner manufacturers use computational models to estimate SAR for specific sequences. These models may vary between scanners, but 3.0T sequences will always have significantly higher SAR compared to sequences run at $1.5 \mathrm{~T}$. Practically, this will require sequences that use high levels of RF power deposition, such as SSFP, to be modified when implementing them at 3.0T to avoid exceeding SAR limits. The most common way to modify a sequence to reduce SAR is to reduce the flip angle, and reduction of flip angle will directly affect SNR. The use of variable-rate selective excitation (VERSE) or other tailored RF pulses can reduce $S A R$ without directly reducing the flip angle [25-28]. Increasing TR will also reduce SAR, but increasing the TR will cause a greater increase in susceptibility artifacts, as will be discussed below.

\section{Changes in Relaxation Times}

Moving from $1.5 \mathrm{~T}$ to $3.0 \mathrm{~T}$ changes the $\mathrm{T} 1$ relaxation time in tissues. $\mathrm{T} 1$ relaxation times at $3.0 \mathrm{~T}$ are generally longer than at $1.5 \mathrm{~T}$. Since the vast majority of CMR sequences have significant T1-weighting, the change in T1 values will affect image contrast and sequence parameters. The $\mathrm{T} 1$ of normal myocardium has been shown to increase between $12 \%$ to $42 \%$ going from $1.5 \mathrm{~T}$ to $3.0 \mathrm{~T}$, and the $\mathrm{T} 1$ of blood has been shown to increase $7 \%$ to $40 \%$ when moving from $1.5 \mathrm{~T}$ to $3.0 \mathrm{~T}[12,29]$. The variation in reported values is probably due to differences in physiology between individuals and differences in pulse sequences and methodologies used to measure $\mathrm{T} 1$.

In the range of $1.5 \mathrm{~T}$ to $3.0 \mathrm{~T}$, the field dependence of $\mathrm{T} 1$ relaxation times for $\mathrm{Gd}$-chelates is quite small [30,31]. Therefore, the $\mathrm{T} 1$ differences between $1.5 \mathrm{~T}$ and 3.0T for blood that exist in the pre-contrast state are negligible early after contrast infusion [12]. The combination of longer T1's in most tissues at 3.0T and the relatively small effects of field strength on Gd-chelate's relaxivity create an advantage for $\mathrm{T} 1$-weighted contrast

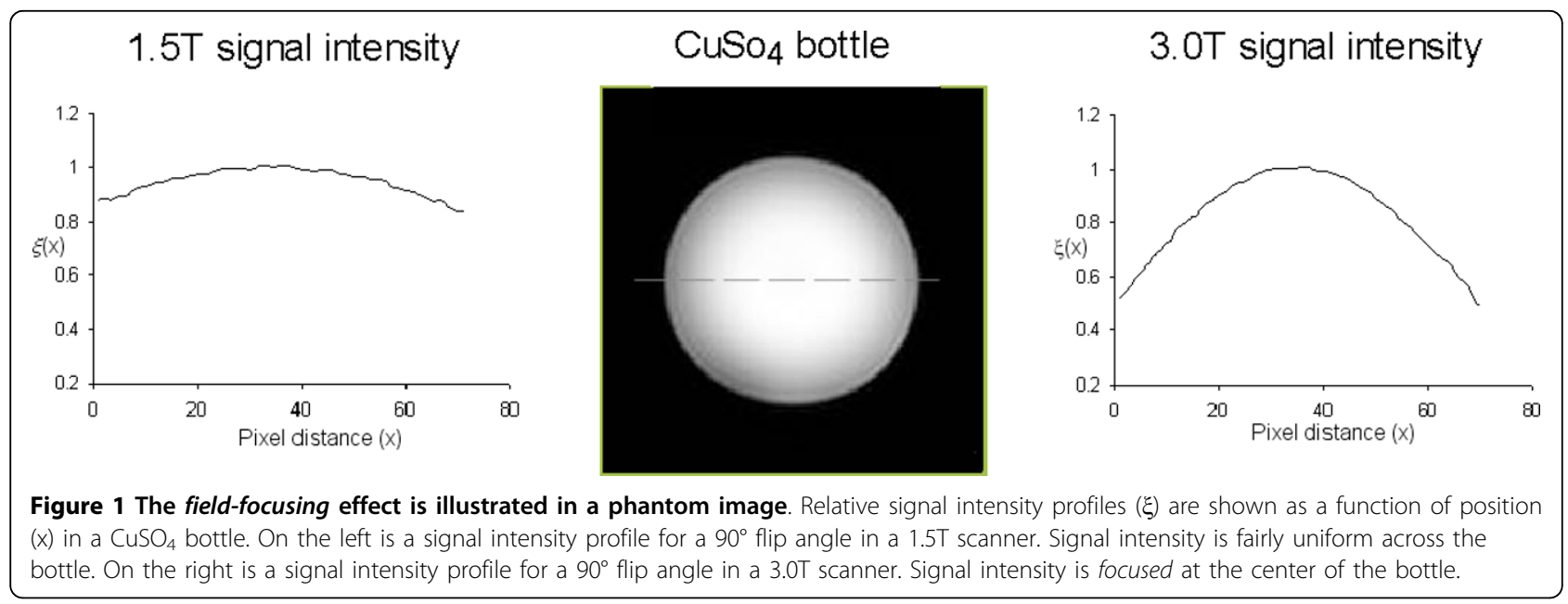


enhanced magnetic resonance angiography (MRA). The longer $\mathrm{T} 1$ in static tissue at 3.0T allows for greater background tissue suppression at $3.0 \mathrm{~T}$ compared to 1.5T. The $\mathrm{T} 1$ reduction in the blood with high levels of Gd-chelates at $3.0 \mathrm{~T}$ is preserved at $1.5 \mathrm{~T}$. This net effect of preserved shortening of blood T1 and improved background suppression is independent of SNR increases; this is one of the factors responsible for the excellent results seen for MRA at 3.0T [32].

Changes in $\mathrm{T} 2$ values at $3.0 \mathrm{~T}$ are negligible for most tissues allowing minimal protocol changes for morphologic T2 spin-echo imaging [29]. However, T2* changes become increasingly significant at $3.0 \mathrm{~T}$ for commonly used gradient-echo cardiac sequences. The reduction in $\mathrm{T} 2$ * going from $1.5 \mathrm{~T}$ to $3.0 \mathrm{~T}$ is a factor of two, suggesting a linear dependence of $\mathrm{T} 2 *$ on field strength. More susceptibility artifacts have been reported with $\mathrm{T} 2 *$ weighted imaging at 3.0T [33]. The reduction of $\mathrm{T} 2 \%$ can be exploited at 3.0T in several ways. First, the blood oxygenation level dependant (BOLD) contrast is based on T2* effects and several studies have shown that the BOLD effect is increased at $3.0 \mathrm{~T}$ compared to $1.5 \mathrm{~T}$ $[34,35]$. Secondly, imaging of iron-based contrast agents has been shown to be more sensitive at $3.0 \mathrm{~T}$ compared to $1.5 \mathrm{~T}$ due to the role of iron on $\mathrm{T} 2 *$ relaxation, (greater iron concentration causes greater regional signal dephasing and signal loss) [34]. Third, T2* sensitivity to iron at 3.0T may be exploited to better delineate individuals with iron-overload as seen in patients with Thalassemia [36,37]. However, the quantification of actual T2* values in areas of very high iron concentration may be more difficult at $3.0 \mathrm{~T}$ due to the very rapid decay of the signal [33].

\section{Field Homogeneity and Susceptibility}

The differences in magnetic susceptibilities of adjacent tissues cause local alterations in the main magnetic field. The magnetic field alterations due to susceptibility differences are a complex function of the local geometry, but are linearly related to the main magnetic field strength [11]. Increasing the magnetic field strength from $1.5 \mathrm{~T}$ to 3.0T causes a linear increase in the susceptibility induced field variations $[38,39]$. These field perturbations will be most prominent at locations where there is a large difference in magnetic susceptibility between tissues, such as the myocardial-lung interface, or areas near the coronary sinus that contain de-oxygenated blood $[39,40]$. The variations in the main magnetic field due to susceptibility differences cause a change in the phase of the MR signal. These local phase changes due to susceptibility differences manifest themselves as signal loss in the image. In a gradient-echo sequence, the phase shift is linearly proportional to the susceptibility difference as well as the echo time (TE). In SSFP sequences, the phase shift is linearly proportional to the susceptibility difference and the repetition time (TR). Since TR > TE, the susceptibility induced signal loss artifacts are more prevalent in SSFP sequences, Figure 2.

The appearance and location of banding artifacts in SSFP images is also affected by field strength and susceptibility differences. The banding artifacts occur at specific frequencies where there is a positive to negative phase transition of the signal. In areas where susceptibility differences cause variations in the main magnetic field, banding artifacts can appear. At 3.0T, the susceptibility effect is greater and hence banding artifacts will be more prevalent. Since the susceptibility differences are proportional to field strength, SSFP sequences at $3.0 \mathrm{~T}$ in areas where tissue susceptibility differences are large are particularly prone to artifacts. For CMR, a prominent location for signal loss and banding artifacts is the infero-lateral wall of the myocardium at the lung/ diaphragm interface.

\section{Results \\ CMR Applications at High Field Strengths Cine Imaging of Cardiac Function}

The use of balanced steady-state free precession (SSFP) imaging has become the cornerstone for cardiac functional analysis with MRI at $1.5 \mathrm{~T}$. SSFP provides significant improvements in SNR and CNR over standard segmented cine gradient-echo methods. At 1.5T, SSFP is less sensitive to inflow and turbulence-related flow voids

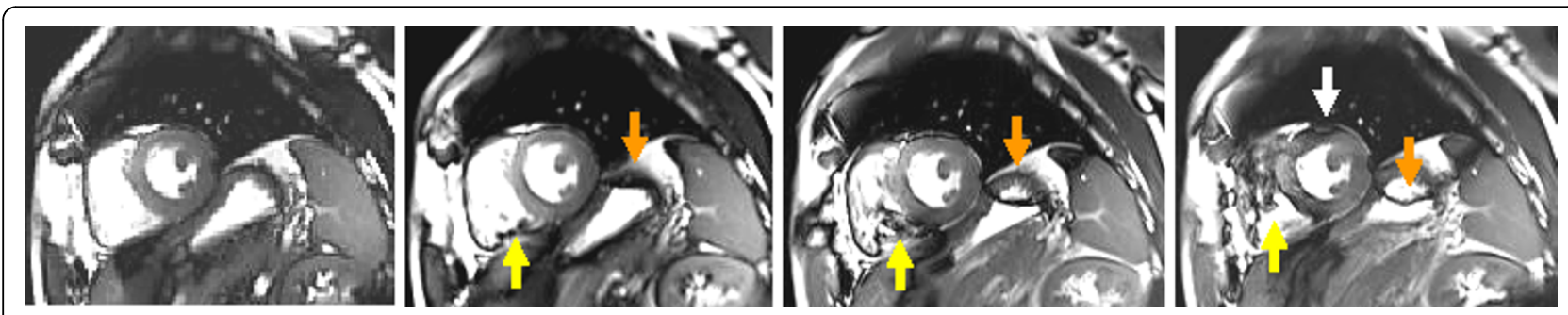

Figure 2 Short-axis, SSFP images acquired at four different repetition times (TR's), ranging from $2.3 \mathrm{msec}$ (far left) to $5.0 \mathrm{msec}$ (far right). As TR is increased, artifacts due to susceptibility and field inhomogeneity are seen in the RV (yellow arrow), at the diaphragm (orange arrow), is and of the anterior and lateral walls of myocardium (white arrows). 
than standard gradient-echo sequences. While implementation of SSFP at 3.0T is feasible with promising results shown by several groups [41-46], it remains an ongoing challenge to overcome the sensitivity of SSFP to high field-related artifacts.

\section{SNR and CNR}

Reproducible image quality for cine SSFP is important for robust evaluation of ventricular function, and with modifications to a 1.5T SSFP sequence, high quality functional cine images can be achieved at 3.0T. In comparison to $1.5 \mathrm{~T}$, cine SSFP has shown increased SNR for myocardium and blood at 3.0T, as well as increased blood-tomyocardium CNR. Increases in CNR have varied widely from $9.4 \%$ to $86 \%$ [41,42]. The differences in reported values are due to differences in acquisition parameters, the extent of shimming, coil positioning, the patient groups studied, and placement of ROI for signal measurements. Due to the potential greater variation in flip angles across the image and the greater effect of susceptibility at $3.0 \mathrm{~T}$, it is important to describe the variability of myocardium SNR segmentally, and between slices.

Comparative analyses of LV function indexes, mass, and volume using cine SSFP has shown no significant difference in values obtained at 3.0T compared to $1.5 \mathrm{~T}$ $[47,48]$. Therefore, one can use existing knowledge and experience of MR quantification algorithms for 3.0T imaging. Compared to 1.5T, CNR between blood and myocardium is reduced in the RV at 3.0T relative to $L V$, which may affect reproducibility of RV functional evaluation [47].

\section{Frequency, Relaxation Time, Homogeneity, and Susceptibility}

One of the most commonly seen artifact in 3.0T SSFP imaging is a "dark-band" artifact described earlier. Dark band locations are related to the local field inhomogeneities and the sequence TR. For similar TR's, heightened local field inhomogeneities at 3.0T may cause the dark bands to come into close proximity of the imaging region-of-interest, potentially resulting in severe image deterioration. Reducing TR widens the band spacing, alleviating the presence of dark bands in the image, insomuch that concurrent increases in bandwidth and SAR are tolerable. A fast, frequency-scout acquisition can be utilized to determine the optimal resonance frequency offset to incorporate with SSFP imaging $[49,50]$. This "frequency scout" offers a visual indication of the resonance offset to be employed, but it requires an additional acquisition, which adds to exam time. A frequency offset based on the frequency scout image, usually on the order of $+/-200 \mathrm{~Hz}$, shifts dark band artifacts away from the imaging region-of-interest. Alternatively, overcoming local field inhomogeneity with a higher-order shim routine offers the clearest benefits to high quality cine SSFP imaging at 3.0T [44]. Use of a shim routine based on a field map of the heart combined with a frequency scout acquisition has shown excellent results. Other methods to address banding artifacts on cine SSFP at 3.0T are being investigated, such as wideband SSFP, which uses sequence adaptation to widen the spacing between dark bands without resonance frequency modification [50].

The use of fast, segmented, spoiled gradient echo (GRE) imaging is prevalent in cardiac imaging, with applications including late gadolinium enhancement imaging, perfusion imaging and MR angiography. After the development of SSFP, use of GRE for functional evaluation has been limited to specific applications, such as quantitative flow imaging and regional detection of flow turbulence and regurgitation. Since the SNR of cine GRE imaging relies predominantly on the steady-state T1 signal of blood and myocardium, there will presumably be reduced SNR at 3.0T for identical pulse sequences due to lengthening of $\mathrm{T} 1$ relaxation times. Although this has been revealed experimentally [41], the resulting contrast-to-noise ratio and overall image quality of GRE has been shown to improve at 3.0T compared to $1.5 \mathrm{~T}$ when using optimized sequences [45]. Cine GRE imaging benefits from its relative insensitivity to field inhomogeneity artifacts in comparison to SSFP. This makes imaging of cardiac function with GRE a reliable option at 3.0T. However, unbalanced gradient rephasing and inflow phenomena may lead to transient signal and flow voids within the ventricle [51]. The use of standard GRE sequences at 3.0T has been examined in the presence of an extra cellular gadolinium-based contrast agent. Hamdan et al showed that long-axis image quality improved after contrast infusion, but short axis images did not improve. The improvement in image quality in the long axis images was due to a reduction in in-plane flow dephasing artifacts. Differences in LV volumes and EF's were also seen between pre-and postcontrast scans. The differences were due to differences in the conspicuity of trabeculae and papillary muscles before and after contrast [51].

\section{Myocardial Tagging}

Myocardial "tagging" is a method in which RF pulses and gradients are used to pre-saturate magnetization perpendicular to the slice plane prior to cine imaging. This pre-excitation pulse suppresses magnetization locally to create "tag lines", so that regional contractile motion can be visualized over the cardiac cycle. Tag lines can be created in a radial, line, or grid pattern. This technique allows the quantification of myocardium strain using computer-assisted programs to track the displacement of tag intersection points over the cardiac cycle. Since the saturated magnetization in the tag line is subject to T1 relaxation over the course of the cardiac cycle, the tag lines gradually fade by diastole. This tag 
fading is a common feature at $1.5 \mathrm{~T}$, since the $\mathrm{T} 1$ of myocardium is approximately $800-900 \mathrm{~ms}$, which is on the order of one cardiac cycle. At 3.0T, this tag fading is lessened since $\mathrm{T} 1$ is longer, allowing tag lines to persist over more of the cardiac cycle. Initial reports have shown improvement of tag line persistence in the LV by $37 \%$ [42]. The tag line persistence is complemented by improved SNR and CNR of the cine gradient echo acquisition. These qualitative improvements at 3.0T translate directly to improved quantitative analysis of myocardial tagging, allowing better detection of tag lines and better depiction of epicardial and endocardial borders for processing multi-directional strain, Figure 3. Implementation of myocardial tagging has also been done using SSFP imaging for signal readout, but application at 3.0T has been limited [52-54].

It is now clear from multiple studies that significant increases in SNR and CNR are gained when performing cine imaging at 3.0T. However, when using SSFP imaging at 3.0T, the presence of artifacts due to susceptibility and banding are more prevalent compared to $1.5 \mathrm{~T}$. Strategies to quickly and easily reduce the artifacts are needed for 3.0T SSFP imaging to completely replace $1.5 \mathrm{~T}$ imaging on a widespread basis.

\section{MR Coronary Angiography}

MR coronary angiography is challenging at any field strength, as the small vessel size and motion of the heart necessitate rapid imaging and high spatial resolution. At $1.5 \mathrm{~T}$, coronary angiography suffers from SNR limitations. Imaging at $3.0 \mathrm{~T}$ could potentially mitigate

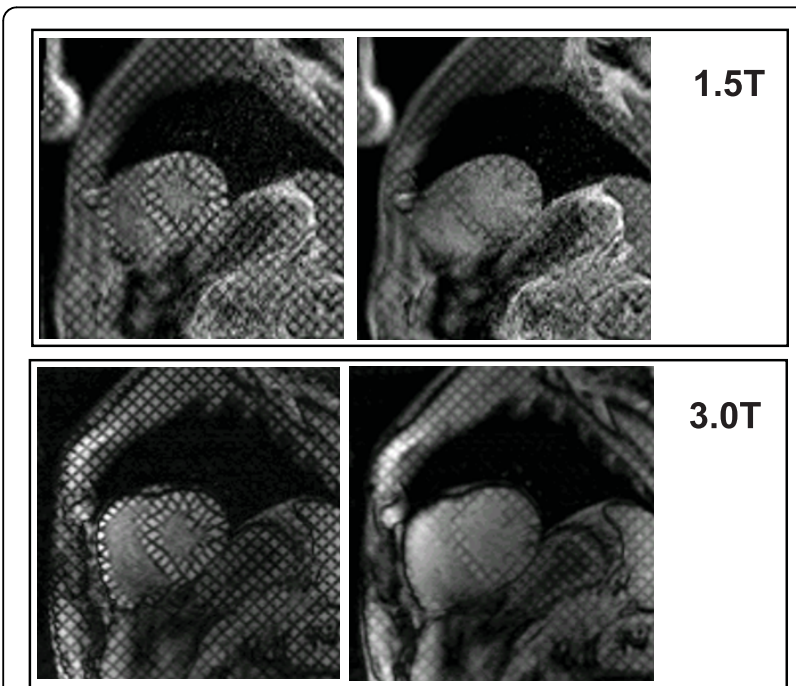

Figure 3 Tagging images acquired in systole and mid-diastole at 3.0T and 1.5T. Note that in diastole tag lines have faded more at $1.5 \mathrm{~T}$ than at 3.0T. The reduced fading at 3.0T is due to the prolonged $\mathrm{T} 1$ at 3.0T. The longer lasting tag lines may allow for better analysis of diastolic function at 3.0T. Signal-to-noise is also higher in the 3.0T images. these SNR concerns. The increased SNR at 3.0T combined with parallel imaging have caused renewed interest in using a $3 \mathrm{D}$ volume that covers the whole heart in a single scan, making it possible to visualize the entire coronary tree [55-57]. Voxel sizes 50\% smaller than those employed at $1.5 \mathrm{~T}$ have been implemented at 3.0T with preserved image quality [58]. Alternately, voxel sizes can be kept at $1.5 \mathrm{~T}$ sizes and the increased SNR available at $3.0 \mathrm{~T}$ can be used solely to reduce image acquisition time with parallel imaging [59].

\section{SNR and CNR}

Several studies have shown that imaging of the coronary arteries is feasible at 3.0T. These studies have been conducted using a variety of different pulse sequences including segmented gradient echo (GRE) sequences $[55,57,58,60,61]$, fast spin echo sequences [62], and recently, SSFP sequences $[63,64]$. There have been two studies directly comparing non-contrast coronary angiography images acquired at 1.5 and 3.0T. Both these studies have demonstrated improved SNR and CNR after moving to $3.0 \mathrm{~T}$. Yang et al reported an average SNR increase of $47 \%$ and a CNR increase of $36 \%$ when moving from 1.5 to $3.0 \mathrm{~T}$ using a high-resolution interleaved spiral GRE sequence [65]. Bi et al reported SNR and CNR increases of $87 \%$ and $83 \%$ respectively when moving from $1.5 \mathrm{~T}$ to $3.0 \mathrm{~T}$ using a high-resolution threedimensional multislab SSFP sequence, although greater image artifacts were also observed at 3.0T [66].

A few reports have examined contrast-enhanced coronary angiography at $3.0 \mathrm{~T}$ although a direct comparison with $1.5 \mathrm{~T}$ has not been done. In a study of 9 healthy volunteers, Bi et al used a three-dimensional, inversion recovery prepared GRE sequence during contrast infusion [55]. A 53\% increase in SNR and a 305\% increase in CNR was observed compared to a comparable non-IR prepared sequence acquired without contrast [55]. In addition, the mean measured length of both the LAD and the RCA was significantly greater in the contrastenhanced images. Kotys et al described a bilateral shadowing artifact along the margins of the coronary arteries when using a high relaxivity contrast agent at 3.0T. Delaying acquisition until the contrast agent has reached steady-state and imaging with the more time efficient centric radial order gave optimal contrast enhancement, but lead to overestimation of the vessel width [67], Figure 4.

\section{Frequency, Relaxation Time, Homogeneity, and Susceptibility}

The greater spectral separation of fat and water may enable more efficient fat-suppression pulses at 3.0T which may improve coronary imaging. Since signal from fatty tissue surrounding the coronaries is often suppressed using a frequency-selective, fat-suppression pulse [68], the greater spectral separation between fat 


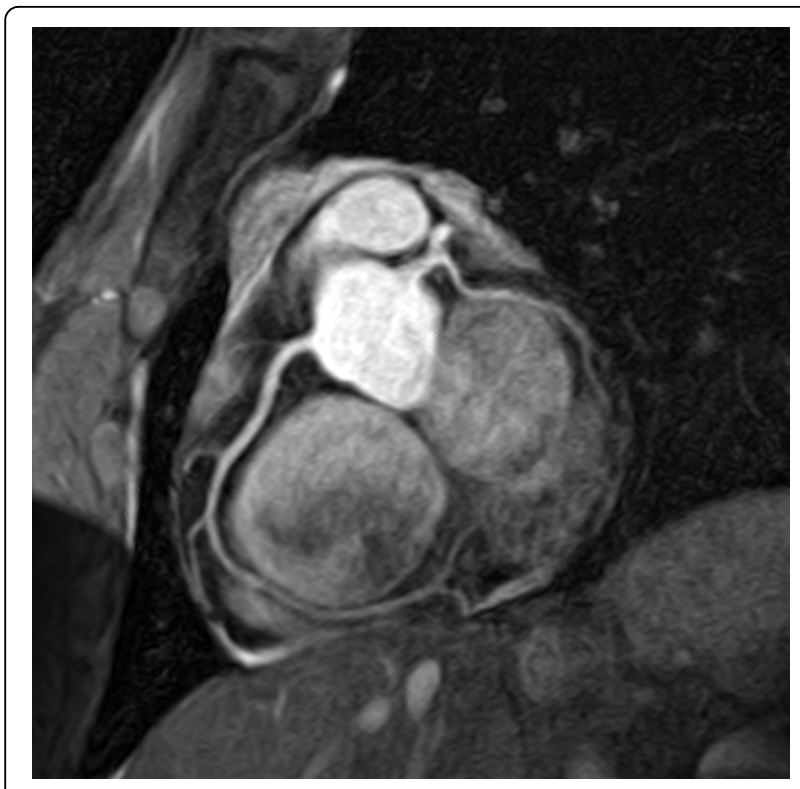

Figure 4 Image of the coronary arteries obtained at 3.0T using a 32-channel coil and employing a targeted 3 D GRE approach. Pixel resolution is $0.7 \times 1.0 \times 2.0$. The right coronary artery (RCA) and left circumflex artery $(\mathrm{LCX})$ and branching vessels can easily be seen. In general, the 3.0T coronary images have superior SNR and CNR compared to $1.5 \mathrm{~T}$.

and water at 3.0T may improve visualization of the coronary arteries by more efficiently suppressing the fat signal around the vessels. Alternately, the better spectral selectivity can be used to shorten the duration of the spectrally-selective contrast preparation pulse. This will reduce sequence $\mathrm{TE}$ and minimize susceptibility artifacts and $\mathrm{T}_{2}{ }^{*}$ signal loss in the images $[59,65]$.

RF field inhomogeneity at $3.0 \mathrm{~T}$ can cause local enhancements in signal intensity in different regions. This local brightening has been observed in coronary MRA scans, depending on the coils used [66]. One possible solution for reducing such signal variation is to use adiabatic RF pulses that are designed to reduce B1 inhomogeneities [66].

Main magnetic field inhomogeneity is more pronounced at $3.0 \mathrm{~T}$ than $1.5 \mathrm{~T}$ especially when imaging over a large volume such as is done in whole heart coronary MRA. Volumetric shimming over the heart can mitigate inhomogeneity effects at 3.0T [68]. To generate greater magnetic field uniformity, a higher order volumetric shim calibration, followed by a dynamic, realtime, multi-slice linear shim to individually fine-tune the shim for each slice before image acquisition can be used [59]. Greater than $40 \%$ reductions in magnetic field inhomogeneities have been demonstrated when higher order shimming was applied (average RMS field deviation decreased from $61.2+/-3.2 \mathrm{~Hz}$ without shimming to $16.5+/-1.3 \mathrm{~Hz}$ when higher-order terms were used) [69].
Susceptibility artifacts increase at 3.0T due to shorter $\mathrm{T} 2 \%$ and greater field inhomogeneities, and these artifacts may obscure coronary vessels along the heart-lung interface [65]. In a direct comparison of MR coronary angiography at 1.5 and $3.0 \mathrm{~T}$, Yang et al noted susceptibility artifacts were present in $9 / 23$ subjects at $3.0 \mathrm{~T}$, but in none of the subjects at $1.5 \mathrm{~T}$ [65]. Use of the shortest $\mathrm{TE}$ and readout gradients possible can help reduce the effects of susceptibility artifacts $[59,65]$. Furthermore, careful shimming can further reduce susceptibility artifacts [56]. It is important to note that although susceptibility artifacts were more prevalent at $3.0 \mathrm{~T}$ in the previously mentioned study, overall image quality for all the coronary segments was actually higher at 3.0T [65].

At $1.5 \mathrm{~T}$, three-dimensional SSFP imaging is the preferred method for the imaging of the coronary arteries [68]. However, SSFP techniques cannot be directly translated to $3.0 \mathrm{~T}$ as the higher frequencies of the RF pulses mean that SAR limits are reached much more rapidly. To implement SSFP sequences at 3.0T, flip angles must be adjusted and/or repetition times increased. Obtaining consistent results when imaging the coronary arteries using SSFP sequences have proved difficult at 3.0T due to the high sensitivity of SSFP to off-resonance artifacts, and it has been suggested that better image quality may be obtained more consistently by using a spoiled GRE sequence instead of SSFP [60,61].

3.0T has caused renewed interest in MR coronary angiography and improvements in coronary imaging have been seen at $3.0 \mathrm{~T}$ compared to $1.5 \mathrm{~T}$. The optimization of coronary imaging at $3.0 \mathrm{~T}$ is continuing.

\section{Myocardial Perfusion}

Myocardial perfusion imaging is critical in determining the extent and location of regional ischemia. Recent studies have shown that myocardial perfusion imaging with MRI employing a gadolinium-based contrast agent is comparable to nuclear based techniques such as SPECT $[64,70]$. Imaging at $3.0 \mathrm{~T}$ offers potential benefits for myocardial perfusion imaging over $1.5 \mathrm{~T}$.

\section{SNR and CNR}

Since myocardial perfusion imaging requires rapid acquisition, SNR and CNR are often compromised at 1.5T. Spatial resolution is often sacrificed for temporal resolution, which can lead to ringing artifacts in the image that can be misinterpreted as perfusion defects [56]. Moving from $1.5 \mathrm{~T}$ to $3.0 \mathrm{~T}$ theoretically produces a doubling of SNR, and therefore perfusion imaging at $3.0 \mathrm{~T}$ may have practical advantages over imaging at 1.5T. The higher SNR at 3.0T can be used to increase either spatial or temporal resolution or can be applied to parallel imaging techniques that decrease image acquisition time [56]. Deciding which is the best choice will depend on the specific application. Better temporal 
resolution is critical in stress perfusion studies, so the added SNR may be best applied to increase the temporal resolution of stress perfusion studies [70]. Longitudinal relaxation time (T1) of myocardial tissue increases with higher field strength and the relaxivity of most gadolinium-based contrast agents does not change greatly with field strength [56]. This translates into potentially increased contrast between perfused and non-perfused myocardial tissue and higher CNR for perfusion images acquired at 3.0 $[56]$

Several studies have reported significant increases in SNR, CNR, and overall image quality for perfusion imaging when moving from 1.5 to $3.0 \mathrm{~T}$ [42,71]. Gutberlet et al reported an SNR increase of $109 \%$ when moving from $1.5 \mathrm{~T}$ to $3.0 \mathrm{~T}$ with a T1-weighted segmented EPI sequence [42]. Aroz et al reported that SNR increased from $25+/-8$ to $82+/-26$ for peak myocardial enhancement during first-pass perfusion imaging when moving from $1.5 \mathrm{~T}$ to $3.0 \mathrm{~T}$ [71]. Plein et al showed that image quality is comparable to $1.5 \mathrm{~T}$ when a five-fold kt-SENSE reduction factor is used at 3.0T [72]. Christian et al showed in an animal study that SNR and CNR were better in perfusion images acquired at 3.0T compared to those acquired at $1.5 \mathrm{~T}$. Correlation between actual flow assessed with microspheres and absolute blood flow assessed by MRI was better at 3.0T than $1.5 \mathrm{~T}$ [73]. However, it is important to note that the SNR and CNR increases observed between 1.5 and 3.0 $\mathrm{T}$ depend on the sequence and specific imaging parameters employed. Diagnostic accuracy of myocardial stress perfusion imaging for detecting hemodynamically relevant coronary artery stenosis at 3.0T has been reported as between 84$86 \%$, which is comparable to previously reported values of $82-89 \%$ at $1.5 \mathrm{~T}$ [74-76]. Diagnostic accuracy of perfusion imaging for detecting myocardial ischemia between 1.5T and 3.0T has not been directly compared.

\section{Frequency, Relaxation Time, Homogeneity, and Susceptibility}

Most centers performing perfusion imaging at $1.5 \mathrm{~T}$ use a non-selective saturation recovery (SR) $90^{\circ} \mathrm{RF}$ pulse rather than an inversion recovery (IR) $180^{\circ} \mathrm{RF}$ pulse to generate contrast between the hypo-perfused and normally-perfused tissue. Although more contrast can theoretically be generated with an IR pulse, the increased speed, reduced heart rate dependence, and more consistent slice to slice contrast have made SR imaging the current standard. Use of SR-based protocols is even more crucial at $3.0 \mathrm{~T}$ compared to $1.5 \mathrm{~T}$. The regional RF inhomogeniety due to field focusing at $3.0 \mathrm{~T}$ will be less evident in a saturation pulse than an inversion pulse. Although the effect can also be seen in a saturation pulse, due to the lower flip angle, the effect will be less significant. Adiabatic $\mathrm{B}_{1}$-insensitive rotation pulses using phase cycling (BIR-4) or pulse trains can be used to increase the spatial homogeneity of the saturation pulse over the heart [56,77]. A recent study has reported successful acquisition of perfusion images at $3.0 \mathrm{~T}$ using a 3 $\mathrm{D}$ gradient echo sequence preceded by a $90^{\circ}$ global adiabatic saturation pulse [78].

The arguments for using a GRE readout versus an SSFP readout are similar to the arguments for using a saturation pre-pulse versus an inversion pre-pulse. Most studies at $1.5 \mathrm{~T}$ are carried out with a GRE readout, although good results have also been obtained using a SSFP readout [79]. The greater susceptibility artifacts of the SSFP at 3.0T make GRE the usual choice for 3.0T perfusion imaging.

Perfusion imaging is playing an increasing role in evaluation of patients with suspected ischemia undergoing cardiac MR exams. Use of a saturation recovery sequence with standard gradient echo readout allows for the minimization of susceptibility artifacts at 3.0T and allows one to take advantage of potential SNR and CNR gains for increased magnetization as well as gains from the increase in T1 of myocardial tissue.

\section{Late Gadolinium Enhancement}

Late Gadolinium enhancement (LGE) imaging is done using an inversion-recovery (IR) prepared gradient echo sequence. The sequence depends on $\mathrm{T} 1$ recovery after the inversion pulse to generate contrast between infarcted myocardium containing gadolinium and normal tissue. Because the sequence generates image contrast based on T1, special attention needs to be paid to timing parameters when performing LGE at 3.0T. As with all sequences at $3.0 \mathrm{~T}$, there are SNR advantages to performing LGE at 3.0T.

\section{SNR and CNR}

Several studies have compared LGE at $1.5 \mathrm{~T}$ and $3.0 \mathrm{~T}$. Klumpp, et al compared 20 subjects imaged at $3.0 \mathrm{~T}$ and a separate set of 20 subjects imaged 1.5T [80]. All subjects had chronic MI and there were no differences between the numbers of segments with LGE in patients imaged at each field strength. A segmented, IR-prepared, GRE sequence was used for imaging, and the inversion time (TI) was optimized for each patient using a TI scout sequence [81]. Imaging parameters were otherwise held constant for the two field strengths, and SNR and CNR were compared. The study found that SNR in the infarcted region at 3.0T was increased 1.6 times compared to $1.5 \mathrm{~T}$, and CNR between normal and infarcted myocardium at $3.0 \mathrm{~T}$ increased 1.9 times when compared to $1.5 \mathrm{~T}$. Huber, et al studied 10 subjects with chronic $\mathrm{MI}$ at both $1.5 \mathrm{~T}$ and 3.0T [82]. A single shot, phase-sensitive inversion recovery (PSIR) sequence was compared at the two field strengths. A TI scout sequence was used to determine the inversion time for each patient. In the magnitude images, CNR between the infarcted and normal myocardium was 2.1 times higher at $3.0 \mathrm{~T}$ compared to $1.5 \mathrm{~T}$. Infarct volume 
between $1.5 \mathrm{~T}$ and 3.0T correlated well but there was some significant variation in individual patients. Bauner et al imaged 15 patients with chronic MI at $1.5 \mathrm{~T}$ and 3.0T [83]. LGE imaging was done with a segmented, IR-prepared, GRE sequence and optimal TI time to null myocardium was determined using a TI scout sequence. SNR of the infarcted myocardium increased by 1.8 times at $3.0 \mathrm{~T}$ compared to $1.5 \mathrm{~T}$. CNR between infarcted and normal myocardium increased by 2 times at $3.0 \mathrm{~T}$ compared to $1.5 \mathrm{~T}$. Infarct volume compared well over all subjects, but BlandAltman analysis showed some significant differences between individual patients. Ligabue et al studied 35 consecutive patients with acute MI at $1.5 \mathrm{~T}$ and 3.0T [84]. LGE imaging was done by an IR-prepared, segmented, GRE sequence and the TI for nulling myocardium was found by a Look-Locker sequence. SNR in infarcted myocardium was increased 3.9 times at $3.0 \mathrm{~T}$ compared to $1.5 \mathrm{~T}$. CNR was increased 3.3 times at $3.0 \mathrm{~T}$ compared to $1.5 \mathrm{~T}$.

These studies all show that there appears to be a significant increase in SNR of infarcted myocardium, and an increase in CNR between normal and infarcted myocardium. This increase in SNR is specifically due to the increase in bulk magnetization going from $1.5 \mathrm{~T}$ to $3.0 \mathrm{~T}$. The majority of studies have used a segmented gradientecho technique for readout. Similar increases are seen using a balanced SSFP readout, but more image artifacts were reported when the SSFP readout was used [82].

\section{Frequency, Relaxation Time, Homogeneity, and Susceptibility}

The infusion of a gadolinium chelate based contrast agent changes the $\mathrm{T} 1$ of the blood and myocardium as a function of the concentration of the contrast agent in the tissue. For the clinical doses used, the relaxation time of the contrast agent dominates over the intrinsic relaxation time of the blood and perfused tissue. Due to the nearly field independent $\mathrm{T} 1$ relaxation values of gadolinium chelates in the $1.5 \mathrm{~T}-3.0 \mathrm{~T}$ range, soon after infusion there is no difference in relaxation times in the blood pool or myocardium at $1.5 \mathrm{~T}$ and $3.0 \mathrm{~T}$ [12]. However, as the contrast agent is cleared from the normal myocardium, the field dependent $\mathrm{T} 1$ difference in the myocardium is again seen. At 15-40 minutes post-contrast infusion, the T1 of normal myocardium which has cleared the majority of the contrast agent is again longer at 3.0T than $1.5 \mathrm{~T}$. This field dependent $\mathrm{T} 1$ contrast will cause the inversion times to null normal myocardium to be longer at 3.0T than at $1.5 \mathrm{~T}$. In a study of subjects with chronic MI, Klumpp et al found that TI times to null normal myocardium were $260 \pm 30 \mathrm{sec}$ at $1.5 \mathrm{~T}$ and $330 \pm 48 \mathrm{sec}$ at $3.0 \mathrm{~T}$ [80]. In a study of patients with acute MI, Ligabue et al found that TI was $330 \pm 50$ at $1.5 \mathrm{~T}$ and $375 \pm 55$ at $3.0 \mathrm{~T}$ [84]. These studies indicate there is a lengthening of the TI time required to null normal myocardium, but the exact difference between $1.5 \mathrm{~T}$ and $3.0 \mathrm{~T}$ will depend on the contrast agent dose and the time after infusion that imaging is conducted, as well as the individual physiology of specific patients. The prolonged T1 of normal myocardium at 3.0T also theoretically increases the available contrast between infarcted and normal myocardium. The increased CNR is partly due greater signal recovery in infarcted myocardium containing the gadolinium chelate based contrast agent when the inversion time for nulling normal myocardium is increased. The amount of this CNR increase will depend on the imaging sequence used, heart rate, and contrast dose, but this effect may contribute to the higher levels of CNR increase seen in LGE imaging at 3.0T.

The RF pulse inhomogeneity affects image quality of LGE at $3.0 \mathrm{~T}$ more than $1.5 \mathrm{~T}$. The variation in the flip angle can cause the inversion pulse to vary across the myocardium and blood pool, potentially yielding a flip angle greater than $180^{\circ}$ in some parts of the heart or blood pool and less than $180^{\circ}$ in other locations. The result of inhomogeneous RF pulses is incomplete suppression of the myocardium, or spatially varying suppression of the myocardium and blood pool. Use of tailored RF pulses or adiabatic inversion pulses reduce this field focusing effect significantly and produce more spatially homogeneous inversion. As mentioned previously, the RF power required to produce an inversion pulse at $3.0 \mathrm{~T}$ is four times greater than the power needed to create the inversion pulse at $1.5 \mathrm{~T}$. This can result in increased RF power deposition in the patient. However, with the long times between inversion pulses in LGE imaging (1-3 heart beats), the increased power seldom causes issues with SAR limitations.

In general, LGE at 3.0T has proven to be as effective as it has been at $1.5 \mathrm{~T}$. SNR as well as infarct-to-myocardium contrast are superior at $3.0 \mathrm{~T}$, and some inherent CNR advantages exist for imaging infarcts at 3.0T. The user must be aware that timing parameters for nulling myocardium will change at 3.0T.

\section{Vessel wall imaging}

Intimal-medial thickness (IMT) has been used as a marker of risk for adverse cardiovascular events and the presence of specific plaque components such as necrotic core has been shown to be important in identifying vulnerable plaques. The reduction or progression of IMT or change in plaque components has been used as an endpoint for judging the efficiency of pharmacologic interventional therapies $[85,86]$. For studies designed to assess changes in vascular wall thickness in response to an intervention, image quality, SNR and CNR are important. Increased SNR, CNR, and image quality lead to better inter-test and inter-observer reproducibility, which allows interventions to be evaluated in a smaller number of subjects, or allows smaller effects to be observed, figure 5 . 
The increased SNR offered by 3.0T has been studied for its effect on increasing reproducibility of vessel wall area, vessel wall volume, and mean wall thickness measurements. In a study of six healthy volunteers, Kokzoglou et al found a 223\% increase in SNR for the carotid wall at 3.0T compared with 1.5T. CNR between the wall and lumen increased $255 \%$. Imaging was done with a multi-slice, black-blood, dual-inversion, turbo spin-echo (TSE) sequence. Both $1.5 \mathrm{~T}$ and $3.0 \mathrm{~T}$ scanners were the same manufacturer, but coil size and imaging parameter varied slightly between field strengths [87]. In a study of 10 healthy volunteers, Dehnavi et al examined the intertest and inter-observer reproducibility of vessel wall area and total lumen area measurements. The relative error between repeated scans was $2.6 \%$ with interclass correlation of 0.98 , a significant improvement over the relative errors seen at $1.5 \mathrm{~T}$ [88]. Similar results were seen in a study by Syed et al that examined 10 subjects at $3.0 \mathrm{~T}$ who had IMT $>0.65 \mathrm{~mm}$ by ultrasound. Subjects were imaged two times using dual-inversion, black-blood, TSE and using a carotid coil. Inter-class correlation was 0.98 for total vessel volume [81]. A study by Yarnykh et al examined five healthy subjects and two patients at $1.5 \mathrm{~T}$ and 3.0T. The 1.5T and 3.0T MR scanners were made by the same manufacturer and coils used were similar. Dual-inversion, black-blood TSE sequences with similar scan parameters were used at both field strengths. SNR increased $77 \pm 44 \%$ for T2-weighted images, and CNR between the wall and lumen increased $82 \pm 46 \%$. No difference between vessel wall area measurements between $1.5 \mathrm{~T}$ and $3.0 \mathrm{~T}$ were noted, suggesting $1.5 \mathrm{~T}$ and $3.0 \mathrm{~T}$ can be used interchangeably in studies [89].

3.0T has also been examined for its ability to improve plaque component identification compared to $1.5 \mathrm{~T}$.

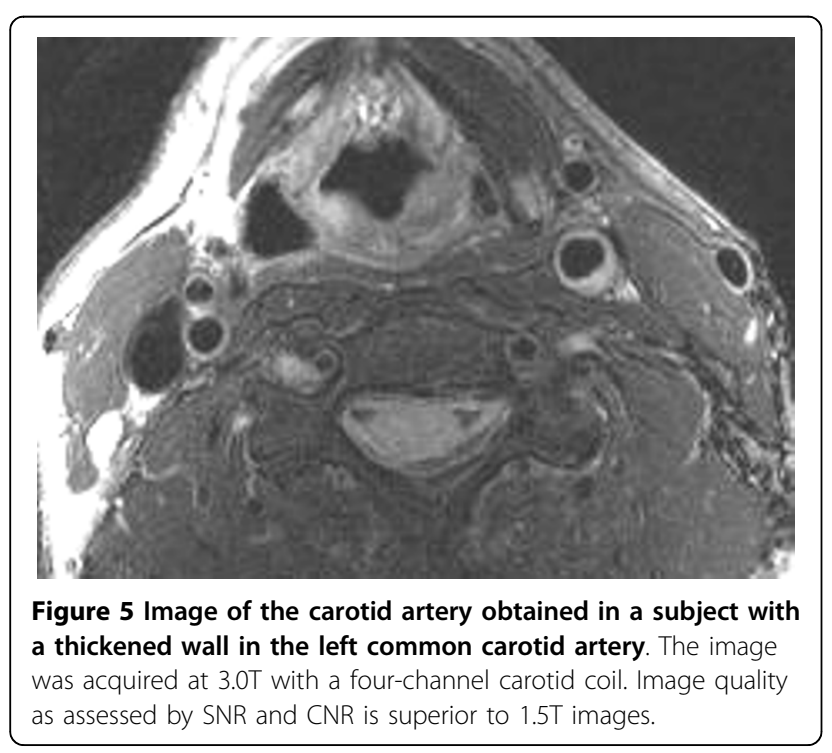

Underhill et al compared the ability of $1.5 \mathrm{~T}$ and $3.0 \mathrm{~T}$ to identify carotid plaque components in 20 subjects with moderate carotid stenoses. Multiple contrast mechanisms were combined to identify plaque components. Importantly, two signal averages were used at $1.5 \mathrm{~T}$, and only a single signal average was used at 3.0T. Despite these differences in number of signal averages, $3.0 \mathrm{~T}$ images showed an increase of SNR of $\approx 20 \%$ over $1.5 \mathrm{~T}$ images. Morphometric variables such as total vessel wall area and lumen agreed well between $1.5 \mathrm{~T}$ and $3.0 \mathrm{~T}$, having inter-class coefficients of 0.88 to 0.96 . This study again suggests $1.5 \mathrm{~T}$ and $3.0 \mathrm{~T}$ can be used interchangeably for morphometric measurements [90].

A study by Vidal et al imaged eight subjects with IMT $\geq 0.5 \mathrm{~mm}$ by ultrasound. Each subject was scanned two times at each field strength, using T2-weighted, dual inversion, black-blood sequences. Both $1.5 \mathrm{~T}$ and $3.0 \mathrm{~T}$ scanners were made by the same manufacturer, and the coils used were virtually identical. The study found a $90 \%$ increase in SNR going from $1.5 \mathrm{~T}$ to $3.0 \mathrm{~T}$ and a $25 \%$ increase in CNR. Although SNR increased, the study found no improvement in reproducibility at $3.0 \mathrm{~T}$ compared to $1.5 \mathrm{~T}$ with the coefficient of variance being $7.8 \%$ and $5.7 \%$ respectively. In addition, this study found that there was a significant difference between vessel wall volumes measured at $1.5 \mathrm{~T}$ compared with $3.0 \mathrm{~T}$. Averaged over all 8 subjects, measurements of vessel wall volume at $1.5 \mathrm{~T}$ were approximately $10 \%$ higher at 1.5T. Data was not presented to assess if this difference was consistent over all subjects [91].

Increased SNR at 3.0T may improve vessel wall imaging in the aorta. In a study of 32 subjects ( 20 healthy volunteers and 12 subjects with cardiac disease), Maroules et al found that SNR increased $\approx 50 \%$ and CNR increased $\approx 70 \%$. Differences in mean wall thickness measurements between $1.5 \mathrm{~T}$ and $3.0 \mathrm{~T}$ were not significant, even with the addition of parallel imaging at 3.0T. Roes et al evaluated navigator-echo gated $3 \mathrm{D}$, dual inversion recovery, black-blood imaging for determining aortic wall thickness [92]. Seven healthy subjects were imaged two times and SNR, CNR, and reproducibility of vessel wall volume were evaluated. The use of the respiratory navigator reduced breathing artifacts as evidenced by an increase in vessel sharpness for the navigator gated sequence over the non-gated sequence. The ICC correlation for inter-scan reproducibility was 0.95 , and the CV was $5.8 \%$.

The increased $\mathrm{T} 1$ values of blood at $3.0 \mathrm{~T}$ compared to $1.5 \mathrm{~T}$ will require a change in the inversion time $(\mathrm{TI})$ in order to null blood. The required changes can be estimated from $\mathrm{T} 1$ measurement of blood at 3.0T.

The use of $3.0 \mathrm{~T}$ for vessel wall imaging in the carotids is an area where there is a clear advantage to 3.0T over $1.5 \mathrm{~T}$. The increase in field strength yields improved 
image quality that translates to improved reproducibility and better identification of plaque components. SAR may be a problem in some black-blood vascular imaging sequences due to the large number of $180^{\circ}$ pulses used.

\section{Conclusion}

Performing CMR studies at 3.0T compared to $1.5 \mathrm{~T}$ causes a number of important physical changes. Foremost among these changes is an increase in bulk magnetization which results in increased SNR. Additionally, changes in the resonant frequency produce increased RF power deposition and an increase in inhomogeneities of the RF excitation field. Changes in the relaxation times require changes in timing parameters that depend on T1. The increase in the main field strength causes an increase in the effect of magnetic susceptibility artifacts.

Several CMR applications have been shown to improve at 3.0T. The majority of improvements are due to the increase in SNR, but some improvements are due to the change in tissue $\mathrm{T} 1$ values. Challenges remain to the widespread use of 3.0T for all cardiac applications. The major limitations are the increased artifacts seen in SSFP imaging and the increased SAR of common sequences. It is important to note that $3.0 \mathrm{~T}$ protocols have not had time to be optimized in the way $1.5 \mathrm{~T}$ protocols have been over the past 10 years. It is clear that simply using $1.5 \mathrm{~T}$ protocol at $3.0 \mathrm{~T}$ may not yield the most optimal results. New developments in parallel imaging, new sequence developments, and further protocol optimization will be required to realize the complete benefits of CMR at 3.0T.

\section{Acknowledgements \\ The authors would like to acknowledge funding from the American Heart Association (Grant-in-aid no. 08553863E), the National Institutes of Health (HL089160), and the Wallace Coulter Foundation.}

\section{Author details}

${ }^{1}$ Department of Radiology, Emory University School of Medicine, 1364 Clifton Road, Room AG34, Atlanta, GA 30322, USA. ²Department of Biomedical Engineering, Emory University and the Georgia Institute of Technology, 101 Woodruff Circle Woodruff Memorial Building, Suite 2001, Atlanta, Georgia 30322, USA. ${ }^{3}$ Laboratory of Integrative Cardiovascular Imaging, Department of Radiology, National Institute of Diabetes and Digestive and Kidney Diseases, National Institutes of Health, Clinical Research Center, Bldg. 10, Rm. 3-5340, MSC 1263, 10 Center Dr., Bethesda, MD 20892, USA.

\section{Authors' contributions}

JNO, JGD, PS, AG, RIP participated in drafting of the manuscript.

\section{Competing interests}

John Oshinski has received research funding from Philips Medical Systems and Siemens Medical Solutions.

Received: 13 August 2010 Accepted: 7 October 2010 Published: 7 October 2010

\section{References}

1. Alvarez-Linera J: 3 T MRI: advances in brain imaging. Eur J Radio/ 2008, 67(3):415-26.
2. DeLano MC, Fisher C: $3 T$ MR imaging of the brain. Magn Reson Imaging Clin N Am 2006, 14(1):77-88.

3. Frayne R, Goodyear BG, Dickhoff P, Lauzon ML, Sevick RJ: Magnetic resonance imaging at 3.0 Tesla: challenges and advantages in clinical neurological imaging. Investigative radiology 2003, 38(7):385-402.

4. Gharib AM, Elagha A, Pettigrew RI: Cardiac magnetic resonance at high field: promises and problems. Curr Probl Diagn Radiol 2008, 37(2):49-56.

5. Lee VS, Hecht EM, Taouli B, Chen Q, Prince K, Oesingmann N: Body and cardiovascular MR imaging at 3.0 T. Radiology 2007, 244(3):692-705.

6. Kuhl CK, Traber F, Gieseke J, Drahanowsky W, Morakkabati-Spitz N, Willinek W, von Falkenhausen M, Manka C, Schild HH: Whole-body highfield-strength (3.0-T) MR imaging in clinical practice. Part II. Technical considerations and clinical applications. Radiology 2008, 247(1):16-35.

7. Kuhl CK, Traber F, Schild HH: Whole-body high-field-strength (3.0-T) MR Imaging in Clinical Practice. Part I. Technical considerations and clinical applications. Radiology 2008, 246(3):675-96.

8. Wieben O, Francois C, Reeder SB: Cardiac MRI of ischemic heart disease at 3 T: potential and challenges. Eur J Radiol 2008, 65(1):15-28.

9. Hoult DI, Phil D: Sensitivity and power deposition in a high-field imaging experiment. J Magn Reson Imaging 2000, 12(1):46-67.

10. Krams R, Wentzel JJ, Oomen JA, Vinke R, Schuurbiers JC, de Feyter PJ, Serruys PW, Slager CJ: Evaluation of endothelial shear stress and $3 \mathrm{D}$ geometry as factors determining the development of atherosclerosis and remodeling in human coronary arteries in vivo. Combining $3 \mathrm{D}$ reconstruction from angiography and IVUS (ANGUS) with computational fluid dynamics. Arterioscler Thromb Vasc Biol 1997, 17(10):2061-5.

11. Haacke EM, Brwon RW, Thonpson MR, Venkatesan R: Magnetic Resonance Imaging: Physical Principles and Sequence Design. John Wiley and Sons 1999.

12. Sharma P, Socolow J, Patel S, Pettigrew RI, Oshinski JN: Effect of Gd-DTPABMA on blood and myocardial T1 at 1.5T and 3T in humans. J Magn Reson Imaging 2006, 23(3):323-30.

13. Wiesinger $F$, Boesiger $P$, Pruessmann KP: Electrodynamics and ultimate SNR in parallel MR imaging. Magn Reson Med 2004, 52(2):376-90.

14. El-Sharkawy AM, Schar M, Ouwerkerk R, Weiss RG, Bottomley PA: Quantitative cardiac 31P spectroscopy at 3 Tesla using adiabatic pulses. Magn Reson Med 2009, 61(4):785-95.

15. Tyler DJ, Emmanuel Y, Cochlin LE, Hudsmith LE, Holloway CJ, Neubauer S, Clarke K, Robson MD: Reproducibility of 31P cardiac magnetic resonance spectroscopy at 3 T. NMR Biomed 2009, 22(4):405-13.

16. Tyler DJ, Hudsmith LE, Clarke K, Neubauer S, Robson MD: A comparison of cardiac (31)P MRS at 1.5 and 3 T. NMR Biomed 2008, 21(8):793-8.

17. Fischbach F, Muller M, Bruhn H: Magnetic resonance imaging of the cranial nerves in the posterior fossa: a comparative study of t2-weighted spin-echo sequences at 1.5 and 3.0 tesla. Acta Radiol 2008, 49(3):358-63.

18. Wen H, Jaffer FA, Denison TJ, Duewell S, Chesnick AS, Balaban RS: The evaluation of dielectric resonators containing $\mathrm{H} 2 \mathrm{O}$ or D2O as RF coils for high-field MR imaging and spectroscopy. J Magn Reson B 1996, 110(2):117-23.

19. Reeder SB, Herzka DA, McVeigh ER: Signal-to-noise ratio behavior of steady-state free precession. Magn Reson Med 2004, 52(1):123-30, PMCID: 2396310.

20. Tyler DD, Butow RA, Gonze J, Estabrook RW: Evidence for the Existence and Function of an Occult, Highly Reactive Sulphydryl Group in the Respiratory Chain Dpnh Dehydrogenase. Biochem Biophys Res Commun 1965, 19:551-7.

21. Hu X, Norris DG: Advances in high-field magnetic resonance imaging. Annu Rev Biomed Eng 2004, 6:157-84.

22. Collins CM, Smith MB: Signal-to-noise ratio and absorbed power as functions of main magnetic field strength, and definition of "90 degrees" RF pulse for the head in the birdcage coil. Magn Reson Med 2001, 45(4):684-91.

23. Vaughan JT, Garwood M, Collins CM, Liu W, DelaBarre L, Adriany G, Andersen $\mathrm{P}$, Merkle $\mathrm{H}$, Goebel R, Smith MB, Uqurbil K: 7T vs. 4T: RF power, homogeneity, and signal-to-noise comparison in head images. Magn Reson Med 2001, 46(1):24-30.

24. Sung K, Nayak KS: Measurement and characterization of RF nonuniformity over the heart at 3T using body coil transmission. J Magn Reson Imaging 2008, 27(3):643-8.

25. Hargreaves BA, Cunningham CH, Nishimura DG, Conolly SM: Variable-rate selective excitation for rapid MRI sequences. Magn Reson Med 2004 52(3):590-7. 
26. Sung K, Nayak KS: Design and use of tailored hard-pulse trains for uniformed saturation of myocardium at 3 Tesla. Magn Reson Med 2008, 60(4):997-1002.

27. Sung K, Nayak KS: B1+ compensation in $3 T$ cardiac imaging using short 2DRF pulses. Magn Reson Med 2008, 59(3):441-6.

28. Kim D, Gonen O, Oesingmann N, Axel L: Comparison of the effectiveness of saturation pulses in the heart at 3T. Magn Reson Med 2008, 59(1):209-15.

29. Stanisz GJ, Odrobina EE, Pun J, Escaravage M, Graham SJ, Bronskill MJ, et al: T1, T2 relaxation and magnetization transfer in tissue at 3T. Magn Reson Med 2005, 54(3):507-12.

30. Rinck PA, Muller RN: Field strength and dose dependence of contrast enhancement by gadolinium-based MR contrast agents. Eur Radiol 1999, 9(5):998-1004.

31. Donahue KM, Burstein D, Manning WJ, Gray ML: Studies of Gd-DTPA relaxivity and proton exchange rates in tissue. Magn Reson Med 1994, 32(1):66-76.

32. Nael K, Fenchel MC, Kramer U, Finn JP, Ruehm SG: Whole-body contrastenhanced magnetic resonance angiography: new advances at 3.0 T. Top Magn Reson Imaging 2007, 18(2):127-34

33. Storey E, Billimoria P: Increased T2 signal in the middle cerebellar peduncles on MRI is not specific for fragile $\times$ premutation syndrome. $J$ Clin Neurosci 2005, 12(1):42-3.

34. Dharmakumar R, Arumana JM, Tang R, Harris K, Zhang Z, Li D: Assessment of regional myocardial oxygenation changes in the presence of coronary artery stenosis with balanced SSFP imaging at 3.0 T: theory and experimental evaluation in canines. J Magn Reson Imaging 2008, 27(5):1037-45.

35. Jahnke C, Gebker R, Manka R, Schnackenburg B, Fleck E, Paetsch I: Navigator-gated $3 \mathrm{D}$ blood oxygen level-dependent CMR at 3.0-T for detection of stress-induced myocardial ischemic reactions. JACC Cardiovasc Imaging 2010, 3(4):375-84.

36. He T, Gatehouse PD, Smith GC, Mohiaddin RH, Pennell DJ, Firmin DN: Myocardial T2* measurements in iron-overloaded thalassemia: An in vivo study to investigate optimal methods of quantification. Magn Reson Med 2008, 60(5):1082-9, PMCID: 2593631.

37. Guo H, Au WY, Cheung JS, Kim D, Jensen JH, Khong PL, Chan Q, Chan KC, Tosti C, Tang H, Brown TR, Lam WW, Ha SY, Brittenham GM, Wu EX: Myocardial T2 quantitation in patients with iron overload at 3 Tesla. J Magn Reson Imaging 2009, 30(2):394-400.

38. Jaffer FA, Wen H, Balaban RS, Wolff SD: A method to improve the B0 homogeneity of the heart in vivo. Magn Reson Med 1996, 36(3):375-83.

39. Reeder SB, Faranesh AZ, Boxerman JL, McVeigh ER: In vivo measurement of $T^{*} 2$ and field inhomogeneity maps in the human heart at 1.5 T. Magn Reson Med 1998, 39(6):988-98, PMCID: 2396319.

40. Atalay MK, Poncelet BP, Kantor HL, Brady TJ, Weisskoff RM: Cardiac susceptibility artifacts arising from the heart-lung interface. Magn Reson Med 2001, 45(2):341-5.

41. Michaely HJ, Nael K, Schoenberg SO, Laub G, Reiser MF, Finn JP, Ruehm SG: Analysis of cardiac function-comparison between 1.5 Tesla and 3.0 Tesla cardiac cine magnetic resonance imaging: preliminary experience. Investigative radiology 2006, 41(2):133-40.

42. Gutberlet $M$, Noeske $R$, Schwinge $K$, Freyhardt $P$, Felix $R$, Niendorf $T$ : Comprehensive cardiac magnetic resonance imaging at 3.0 Tesla: feasibility and implications for clinical applications. Invest Radiol 2006, 41(2):154-67.

43. Gutberlet M, Schwinge K, Freyhardt P, Spors B, Grothoff M, Denecke T, Lüdemann $L$, Noeske R, Niendorf T, Felix R: Influence of high magnetic field strengths and parallel acquisition strategies on image quality in cardiac 2 D CINE magnetic resonance imaging: comparison of $1.5 \mathrm{~T}$ vs. 3.0 T. Eur Radiol 2005, 15(8):1586-97.

44. Schar M, Kozerke S, Fischer SE, Boesiger P: Cardiac SSFP imaging at 3 Tesla. Magn Reson Med 2004, 51(4):799-806

45. Tyler DJ, Hudsmith LE, Petersen SE, Francis JM, Weale P, Neubauer S, Clarke K, Robson MD: Cardiac cine MR-imaging at 3T: FLASH vs SSFP. J Cardiovasc Magn Reson 2006, 8(5):709-15.

46. Wintersperger BJ, Bauner K, Reeder SB, Friedrich D, Dietrich O, Sprung KC, Picciolo M, Nikolaou K, Reiser MF, Schoenberg SO: Cardiac steady-state free precession CINE magnetic resonance imaging at 3.0 tesla: impact of parallel imaging acceleration on volumetric accuracy and signal parameters. Investigative radiology 2006, 41(2):141-7.
47. Hudsmith LE, Cheng AS, Tyler DJ, Shirodaria C, Lee J, Petersen SE, Francis JM, Clarke K, Robson MD, Neubauer S: Assessment of left atrial volumes at 1.5 Tesla and 3 Tesla using FLASH and SSFP cine imaging. J Cardiovasc Magn Reson 2007, 9(4):673-9.

48. Maroules CD, McColl R, Khera A, Peshock RM: Interstudy reproducibility of SSFP cine magnetic resonance: impact of magnetic field strength and parallel imaging. J Magn Reson Imaging 2008, 27(5):1139-45.

49. Deshpande VS, Shea SM, Li D: Artifact reduction in true-FISP imaging of the coronary arteries by adjusting imaging frequency. Magn Reson Med 2003, 49(5):803-9.

50. Nayak KS, Lee HL, Hargreaves BA, Hu BS: Wideband SSFP: alternating repetition time balanced steady state free precession with increased band spacing. Magn Reson Med 2007, 58(5):931-8.

51. Hamdan A, Kelle S, Schnackenburg B, Fleck E, Nagel E: Improved quantitative assessment of left ventricular volumes using TGrE approach after application of extracellular contrast agent at 3 Tesla. J Cardiovasc Magn Reson 2007, 9(6):845-53.

52. Zwanenburg JJ, Kuijer JP, Marcus JT, Heethaar RM: Steady-state free precession with myocardial tagging: CSPAMM in a single breathhold. Magn Reson Med 2003, 49(4):722-30.

53. Markl M, Scherer S, Frydrychowicz A, Burger D, Geibel A, Hennig J: Balanced left ventricular myocardial SSFP-tagging at 1.5T and 3T. Magn Reson Med 2008, 60(3):631-9.

54. Ibrahim el SH, Stuber M, Schar M, Osman NF: Improved myocardial tagging contrast in cine balanced SSFP images. J Magn Reson Imaging 2006, 24(5):1159-67.

55. Bi X, Li D: Coronary arteries at $3.0 \mathrm{~T}$ : Contrast-enhanced magnetizationprepared three-dimensional breathhold MR angiography. J Magn Reson Imaging 2005, 21(2):133-9.

56. Fenchel M, Kramer U, Nael K, Miller S: Cardiac magnetic resonance imaging at 3.0 T. Top Magn Reson Imaging 2007, 18(2):95-104.

57. Huber ME, Kozerke S, Pruessmann KP, Smink J, Boesiger P: Sensitivityencoded coronary MRA at 3T. Magn Reson Med 2004, 52(2):221-7.

58. Stuber M, Botnar RM, Fischer SE, Lamerichs R, Smink J, Harvey $P$, Manning WJ: Preliminary report on in vivo coronary MRA at 3 Tesla in humans. Magn Reson Med 2002, 48(3):425-9.

59. Santos JM, Cunningham CH, Lustig M, Hargreaves BA, Hu BS, Nishimura DG, Pauly JM: Single breath-hold whole-heart MRA using variable-density spirals at 3T. Magn Reson Med 2006, 55(2):371-9.

60. Kaul MG, Stork A, Bansmann PM, Nolte-Ernsting C, Lund GK, Weber C, Adam G: Evaluation of balanced steady-state free precession (TrueFISP) and K-space segmented gradient echo sequences for $3 \mathrm{D}$ coronary MR angiography with navigator gating at 3 Tesla. Rofo 2004, 176(11): 1560-5.

61. Priest AN, Bansmann PM, Mullerleile K, Adam G: Coronary vessel-wall and lumen imaging using radial k-space acquisition with MRI at 3 Tesla. Eur Radiol 2007, 17(2):339-46.

62. Koktzoglou I, Simonetti O, Li D: Coronary artery wall imaging: initial experience at 3 Tesla. J Magn Reson Imaging 2005, 21(2):128-32.

63. Liu X, Bi X, Huang J, Jerecic R, Carr J, Li D: Contrast-enhanced whole-heart coronary magnetic resonance angiography at $3.0 \mathrm{~T}$ : comparison with steady-state free precession technique at $1.5 \mathrm{~T}$. Investigative radiology 2008, 43(9):663-8.

64. Wagner A, Mahrholdt H, Sechtem U, Kim RJ, Judd RM: MR imaging of myocardial perfusion and viability. Magn Reson Imaging Clin N Am 2003, 11(1):49-66.

65. Yang PC, Nguyen P, Shimakawa A, Brittain J, Pauly J, Nishimura D, Hu B, McConnell M: Spiral magnetic resonance coronary angiography-direct comparison of 1.5 Tesla vs. 3 Tesla. J Cardiovasc Magn Reson 2004, 6(4):877-84.

66. Bi X, Deshpande V, Simonetti O, Laub G, Li D: Three-dimensional breathhold SSFP coronary MRA: a comparison between 1.5T and 3.0T. J Magn Reson Imaging 2005, 22(2):206-12

67. Kotys MS, Herzka DA, Vonken EJ, Ohayon J, Heroux J, Gharib AM, Stuber M, Pettigrew Rl: Profile order and time-dependent artifacts in contrastenhanced coronary MR angiography at 3T: origin and prevention. Magn Reson Med 2009, 62(2):292-9.

68. Nael K, Fenchel M, Saleh R, Finn JP: Cardiac MR imaging: new advances and role of 3T. Magn Reson Imaging Clin N Am 2007, 15(3):291-300, v.

69. Kim DH, Adalsteinsson E, Glover GH, Spielman DM: Regularized higherorder in vivo shimming. Magn Reson Med 2002, 48(4):715-22. 
70. Gerber BL, Raman SV, Nayak K, Epstein FH, Ferreira P, Axel L, Kraitchman DL: Myocardial first-pass perfusion cardiovascular magnetic resonance: history, theory, and current state of the art. J Cardiovasc Magn Reson 2008, 10(1):18.

71. Araoz PA, Glockner JF, McGee KP, Potter DD, Valeti VU, Stanley DW, Christian TF: 3 Tesla MR imaging provides improved contrast in first-pass myocardial perfusion imaging over a range of gadolinium doses. $J$ Cardiovasc Magn Reson 2005, 7(3):559-64.

72. Plein S, Schwitter J, Suerder D, Greenwood JP, Boesiger P, Kozerke S: kSpace and time sensitivity encoding-accelerated myocardial perfusion MR imaging at 3.0 T: comparison with 1.5 T. Radiology 2008, 249(2):493-500.

73. Christian TF, Bell SP, Whitesell $L$, Jerosch-Herold M: Accuracy of cardiac magnetic resonance of absolute myocardial blood flow with a high-field system: comparison with conventional field strength. JACC CardiovasC Imaging 2009, 2(9):1103-10.

74. Wolff SD, Schwitter J, Coulden R, Friedrich MG, Bluemke DA, Biederman RW, Martin ET, Lansky AJ, Kashanian F, Foo TK, Licato PE, Comeau CR: Myocardial first-pass perfusion magnetic resonance imaging: a multicenter dose-ranging study. Circulation 2004, 110(6):732-7.

75. Gebker R, Jahnke C, Paetsch I, Kelle S, Schnackenburg B, Fleck E, Nagel E: Diagnostic performance of myocardial perfusion MR at $3 \mathrm{~T}$ in patients with coronary artery disease. Radiology 2008, 247(1):57-63.

76. Nagel E, Lehmkuhl HB, Bocksch W, Klein C, Vogel U, Frantz E, Ellmer A, Dreysse $\mathrm{S}$, Fleck E: Noninvasive diagnosis of ischemia-induced wall motion abnormalities with the use of high-dose dobutamine stress MRI: comparison with dobutamine stress echocardiography. Circulation 1999, 99(6):763-70.

77. Kim D, Cernicanu A, Axel L: B(0) and B(1)-insensitive uniform $T(1)$ weighting for quantitative, first-pass myocardial perfusion magnetic resonance imaging. Magn Reson Med 2005, 54(6):1423-9.

78. Shin $\mathrm{T}, \mathrm{Hu} H \mathrm{HH}$, Pohost GM, Nayak KS: Three dimensional first-pass myocardial perfusion imaging at 3T: feasibility study. J Cardiovasc Magn Reson 2008, 10(1):57

79. Lyne JC, Gatehouse PD, Assomull RG, Smith GC, Kellman P, Firmin DN, Pennell DJ: Direct comparison of myocardial perfusion cardiovascular magnetic resonance sequences with parallel acquisition. J Magn Reson Imaging 2007, 26(6):1444-51.

80. Klumpp B, Fenchel M, Hoevelborn T, Helber U, Scheule A, Claussen C, Miller S: Assessment of myocardial viability using delayed enhancement magnetic resonance imaging at 3.0 Tesla. Investigative radiology 2006, 41(9):661-7.

81. Syed MA, Oshinski JN, Kitchen C, Ali A, Charnigo RJ, Quyyumi AA: Variability of carotid artery measurements on 3-Tesla MRI and its impact on sample size calculation for clinical research. Int J Cardiovasc Imaging 2009, 25(6):581-9.

82. Huber A, Bauner K, Wintersperger BJ, Reeder SB, Stadie F, Mueller E, Schmidt M, Winnik E, Reiser MF, Schoenberg : Phase-sensitive inversion recovery (PSIR) single-shot TrueFISP for assessment of myocardial infarction at 3 tesla. Investigative radiology 2006, 41(2):148-53.

83. Bauner KU, Muehling O, Wintersperger BJ, Winnik E, Reiser MF, Huber A: Inversion recovery single-shot TurboFLASH for assessment of myocardial infarction at 3 Tesla. Investigative radiology 2007, 42(6):361-71.

84. Ligabue G, Fiocchi F, Ferraresi S, Barbieri A, Rossi R, Modena MG, Romagnoli R, Torricelli P: 3-Tesla MRI for the evaluation of myocardial viability: a comparative study with 1.5-Tesla MRI. Radiol Med 2008, 113(3):347-62.

85. de Groot E, van Leuven SI, Duivenvoorden R, Meuwese MC, Akdim F, Bots ML, Kastelein JJ: Measurement of carotid intima-media thickness to assess progression and regression of atherosclerosis. Nat Clin Pract Cardiovasc Med 2008, 5(5):280-8.

86. Taylor A, Shaw LJ, Fayad Z, O'Leary D, Brown BG, Nissen S, Rader D, Raggi P: Tracking atherosclerosis regression: a clinical tool in preventive cardiology. Atherosclerosis 2005, 180(1):1-10.

87. Koktzoglou I, Chung YC, Mani V, Carroll TJ, Morasch MD, Mizsei G, Simonetti OP, Fayad ZA, Li D: Multislice dark-blood carotid artery wall imaging: a 1.5 T and 3.0 T comparison. J Magn Reson Imaging 2006, 23(5):699-705.

88. Alizadeh Dehnavi R, Doornbos J, Tamsma JT, Stuber M, Putter H, van der Geest RJ, Lamb HJ, de Roos A: Assessment of the carotid artery by MRI at 3T: a study on reproducibility. J Magn Reson Imaging 2007, 25(5):1035-43.
89. Yarnykh VL, Terashima M, Hayes CE, Shimakawa A, Takaya N, Nguyen PK, Brittain JH, McConnell MV, Yuan C: Multicontrast black-blood MRI of carotid arteries: comparison between 1.5 and 3 tesla magnetic field strengths. J Magn Reson Imaging 2006, 23(5):691-8.

90. Underhill HR, Yuan C, Terry JG, Chen H, Espeland MA, Hatsukami TS, Saam T, Chu B, Yu W, Oikawa M, Takaya N, Yarnykh VL, Kraft R, Carr JJ, Maldjian J, Tang R, Crouse JR: Differences in carotid arterial morphology and composition between individuals with and without obstructive coronary artery disease: a cardiovascular magnetic resonance study. J Cardiovasc Magn Reson 2008, 10(1):31, PMCID: 2440371.

91. Vidal A, Bureau Y, Wade T, Spence JD, Rutt BK, Fenster A, Parraga G: Scanrescan and intra-observer variability of magnetic resonance imaging of carotid atherosclerosis at 1.5 T and 3.0 T. Phys Med Biol 2008, 53(23):6821-35.

92. Maroules CD, McColl R, Khera A, Peshock RM: Assessment and reproducibility of aortic atherosclerosis magnetic resonance imaging: impact of 3-Tesla field strength and parallel imaging. Investigative radiology 2008, 43(9):656-62.

doi:10.1186/1532-429X-12-55

Cite this article as: Oshinski et al:: Cardiovascular magnetic resonance at 3.0T: Current state of the art. Journal of Cardiovascular Magnetic Resonance 2010 12:55.

\section{Submit your next manuscript to BioMed Central and take full advantage of:}

- Convenient online submission

- Thorough peer review

- No space constraints or color figure charges

- Immediate publication on acceptance

- Inclusion in PubMed, CAS, Scopus and Google Scholar

- Research which is freely available for redistribution

Submit your manuscript at www.biomedcentral.com/submit
C Biomed Central 